\title{
Hyaluronic Acid-based Hydrating Vaginal Gel
}

National Cancer Institute

\section{Source}

National Cancer Institute. Hyaluronic Acid-based Hydrating Vaginal Gel. NCI Thesaurus.

Code C102536.

A clear, colorless water-based vaginal gel containing the partial benzyl ester of hyaluronic acid (HA) with potential hydrating activity. Upon vaginal application, $\mathrm{HA}$ adheres to the vaginal mucosa where it retains water, provides moisture to the vagina and protects the vaginal mucosa. This gel may provide relief in vaginal dryness and may prevent sexual discomfort, itching and irritation. This HA derivative is less susceptible to enzymatic breakdown and provides longer lasting activity compared to HA. HA is naturally present in the vaginal epithelium. 